\title{
A Comparison of Discrete Element and Micromechanical Methods for Determining the Effective Elastic Properties of Geomaterials
}

DOI:

10.1016/j.compgeo.2017.01.018

\section{Document Version}

Accepted author manuscript

Link to publication record in Manchester Research Explorer

Citation for published version (APA):

Geer, S., Berger, J. R., Parnell, W., \& Mustoe, G. G. W. (2017). A Comparison of Discrete Element and Micromechanical Methods for Determining the Effective Elastic Properties of Geomaterials. Computers and Geotechnics, 87, 1-9. https://doi.org/10.1016/j.compgeo.2017.01.018

Published in:

Computers and Geotechnics

\section{Citing this paper}

Please note that where the full-text provided on Manchester Research Explorer is the Author Accepted Manuscript or Proof version this may differ from the final Published version. If citing, it is advised that you check and use the publisher's definitive version.

\section{General rights}

Copyright and moral rights for the publications made accessible in the Research Explorer are retained by the authors and/or other copyright owners and it is a condition of accessing publications that users recognise and abide by the legal requirements associated with these rights.

\section{Takedown policy}

If you believe that this document breaches copyright please refer to the University of Manchester's Takedown Procedures [http://man.ac.uk/04Y6Bo] or contact uml.scholarlycommunications@manchester.ac.uk providing relevant details, so we can investigate your claim.

\section{OPEN ACCESS}




\title{
A Comparison of Discrete Element and Micromechanical Methods for Determining the Effective Elastic Properties of Geomaterials
}

\author{
S. Geer ${ }^{\mathrm{a}}$, J. R. Berger ${ }^{\mathrm{b}}$, William J. Parnell ${ }^{\mathrm{c}}$, G. G. W. Mustoe ${ }^{\mathrm{b}}$ \\ ${ }^{a}$ Department of Civil Engineering, University of Arkansas, Fayetville, \\ Arkansas, USA \\ ${ }^{b}$ Department of Mechanical Engineering, Colorado School of Mines, Golden, \\ Colorado, USA \\ ${ }^{c}$ School of Mathematics, University of Manchester, Oxford Road, \\ Manchester, M13 9PL, UK
}

\begin{abstract}
Data generated from scanning electron microscopy images of oil-shale geomaterials are used to generate configurations and to acquire parameters required for use in homogenization schemes for the determination of the effective elastic properties of the samples. Two alternative homogenization methods are employed: numerical simulation using the Discrete Element Method and the Polycrystalline Self-Consistent Method from micromechanics. The schemes give rise to predictions of the effective elastic properties that are in very good agreement.
\end{abstract}

Keywords: Homogenization, Discrete Element Method, Micromechanics, Geomaterials, Polycrystalline Self-Consistent Method, Anisotropy, Stiffness Tensor, Hill Tensor, Concentration Tensor

\section{Introduction}

Geomaterials possess all manner of heterogeneity at a number of lengthscales. Estimating the mechanical properties of such media has therefore attracted a great deal of attention over the years, e.g. [1]. On the large scale, one can visually detect heterogeneities and discontinuities, i.e. bedding planes, faults, pores and cracks for example. Similarly, inhomogeneity 
is present at the microscale, although often not detectable by the human eye. Such microscale inhomogeneities include those types mentioned at the larger scale but also micropores, microcracks, mineral grains and crystals, to name but a few. These inhomogeneities will in general induce strong anisotropy in the material. A common approach is to attempt to define a so-called representative volume element (RVE) containing a large number of microscale inhomogeneities, distributed in a statistically homogeneous manner. This enables effective, homogeneous, anisotropic properties to be defined associated with the RVE or sample, in question [2] and [3]. This so-called homogenization process is particularly useful when loading conditions are such that fields induced in the sample vary on a length scale much larger than any inhomogeneity length scale. It is also important to note that homogenization can be applied at a so-called mesoscale if one wishes, so that large scale heterogeneity can be incorporated into field models if required [4]. Here, two methods are employed in order to estimate the effective homogeneous anisotropic elastic properties of oil-shale geomaterials where the side of the RVE in question is 20mm: the Discrete Element Method (DEM) and the Polycrystalline Self-Consistent Method (PSCM).

The DEM is used in a wide variety of engineering problems in order to efficiently model discontinuous and continuous media, including problems involving powders and rock mechanics [5] and [6]. In [7], a two dimensional (2D) DEM was employed in order to estimate the elastic properties of layered geomaterials. DEM systems of approximately 16, 000 particles were created, settled, and bonded, and different particle stiffness properties were assigned, based on their location in the DEM system, to represent a petroleum-bearing geomaterial comprised of a mineral and a kerogen component. Effective elastic properties were estimated using the DEM when the sample is subjected to displacement boundary conditions and the medium is approximated as transversely isotropic. Parameter studies were carried out on bonded materials, varying kerogen content, confining pressure, porosity, particle resolution, and layer geometry. These studies determined that all parameters, except for confining pressure, have a significant influence on the effective elastic properties of the samples. [8] establish upper and lower estimates of the elastic moduli for granular materials and relate a micromechanical description of strain to the packing structure. [9] suggests the elastic response of a granular material is dependent on the coordination number, which is linked to the fluctuation of the number of contacts per particle in a granular system subjected to shear strain. [10] developed used microstructure to develop expressions for 
the elastic constants of granular assemblies with linear contact interactions. [11] shows that, if a 2D system of particles can be considered a homogeneous 2D material, the macroscopic stress-strain behavior can be described by 4 independent elastic moduli.

Micromechanical methods to predict the effective elastic properties of inhomogeneous media, see for example [3] date back to the pioneering work of [12] and [13] but the theory was developed significantly in the 1960s due to the associated need to understand composite materials that were being developed at that time. Assuming that an RVE has been identified, standard methods give rise to predictions of the effective elastic properties in terms of so-called concentration tensors, linking fields inside certain phases of the medium to the imposed field. Many methods employ techniques associated with the canonical inhomogeneity problem and exploit the useful simplifications that occur for ellipsoidal inhomogeneities giving rise to the useful Eshelby $\mathbf{S}$-tensor and Hill $\mathbf{P}$-tensor [14]. The simplest such scheme is the so-called dilute estimate (DE), based on the seminal work by [15] where interactions between phases are neglected. This method is computationally cheap, explicit, and simple to implement; however, it has been found to have only reasonable accuracy when the volume fraction of inclusion(s) in a host material is small. Extensions to non-dilute volume fractions are numerous but perhaps the most popular include the Mori-Tanaka [16], differential [17], self-consistent [18], [19], [20] and [21] and generalized self-consistent methods [22]. A variety of self-consistent methods have been proposed. It appears that for geomaterials, the so-called Polycrystalline self-consistent method (PSCM) is most appropriate since generally no host medium can be discerned.

Finite element methods have been used in conjunction with Monte Carlo simulations by a number of authors to determine the effect of randomly distributed microstructure on the effective elastic properties of heterogeneous materials. In [23] randomly distributed voids were considered and it was concluded that the predictions were in good agreement with analytic and alternative numerical models. [24] and [25] used similar approaches to obtain estimates of effective properties.

Here, data is acquired from scanning electron microscopy (SEM) images of oil-shale samples in order to generate two dimensional configurations that can be used in the DEM and PSCM. In the case of the DEM a virtual model of a rock sample is created that represents the original rock sample on the microscale both in terms of geometry and mineralogy. Random-pack systems of circular particles are generated in the RVE domain and assigned 
mineralogical properties based on the SEM data, restricted to six mineral types. Volume fraction data for each of the six mineral phases are also acquired. These particles are then bonded using the parallel bonding algorithm of [5] before the DEM simulations are performed. Two different displacement boundary condition states are then imposed on samples in order to determine the effective elastic properties. For the implementation of the PSCM the standard micromechanical expressions are employed for circular cylinders, for a six-phase medium with volume fractions assigned as those acquired from the images referred to above. Comparisons reveal that both methods give good agreement.

Subscript notation is mainly used in the paper, where the summation convention is implied over repeated indices and the range of the subscripts is 1 to 3 unless otherwise indicated. In the absence of subscripts, lowercase boldface for vectors (e.g., a) is used.

\section{Geomaterials and DEM}

Oil shale samples provided courtesy of the Center for Oil Shale Technology and Research (COSTAR) at the Colorado School of Mines in Golden, $\mathrm{CO}$, are extracted from the Eocene geologic formation known as the Green River Formation and analyzed using Quantitative Evaluation of Minerals by Scanning Electron Microscopy, or QEMSCAN. More details about this quantitative method can be found in [26]. Details concerning the preparation of the samples prior to QEMSCAN analysis can be found in [27]. Two different QEMSCAN analyses are performed on each of three physical samples. One analysis yielded a two-species representation of the material comprised of pores and solid materials, and the second analysis yielded a multiple-species representation of the sample comprised of various minerals.

\subsection{RVE Configurations For Homogenization Derived From Imaging}

For the DEM-based analyses of the geomaterial data, random pack (RP) systems of particles are generated, settled, and bonded in a square domain. The particles in the 2D systems are assigned mineralogical identities using hashing techniques; this involves converting the geomaterial images into text files using the image processing software Image $J$, discussed in [28]. An example of this conversion is shown in Figure 1, in which a simple 8-bit, red/green/blue (RGB) 256 by 256 pixel image is converted to a text file; a portion of the text file is displayed in order to show that geometry and color 
data are preserved during the conversion. Only two different integers identify the colors of the pixels in the black and white image shown on the left side of Figure 1; a value of 0 represents black, and a value of 255 represents white.

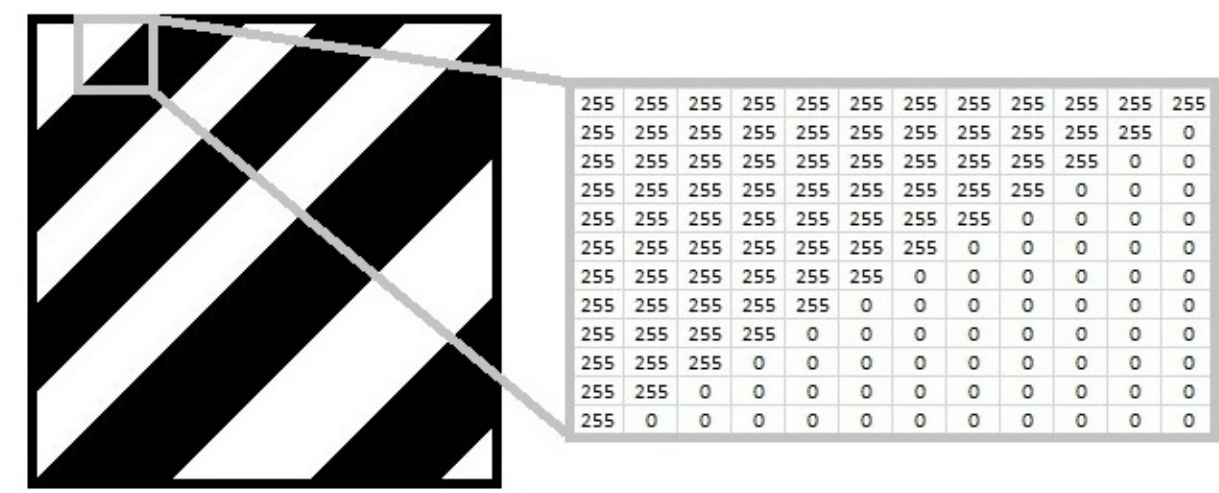

Figure 1: An example of the conversion of an image to a text file.

Integrating the information contained in the text files into DEM simulations is achieved by discretizing the domain in which the DEM particles are generated and subsequently settled into a number of regions consistent with the number of pixels in the geomaterial images. Figure 2 (left) shows a representation of a 4 by 4 pixel image of an object containing two materials, identified each by integers 255 and 0; a DEM particle system, Figure 2 (right) has been created with randomly-distributed particles in a square region of virtual space. The image data can be superimposed over the DEM system region by setting the points, in Cartesian coordinates, defining both regions to be coincident, i.e. $\left(P_{x 1}, P_{y 1}\right)=\left(P_{x 3}, P_{y 3}\right)$ and $\left(P_{x 2}, P_{y 2}\right)=\left(P_{x 4}, P_{y 4}\right)$. The dimensions of the grid spaces, $\Delta_{x}$ and $\Delta_{y}$, are found by,

$$
\begin{aligned}
\Delta_{x} & =\frac{P_{x 2}-P_{x 1}}{N_{x}} \\
\Delta_{y} & =\frac{P_{y 2}-P_{y 1}}{N_{y}},
\end{aligned}
$$

where $N_{x}$ and $N_{y}$ are the number of pixels in the $x$ and $y$-directions, respectively. A particle whose centroid falls within a text image grid containing a certain integer value is flagged with that same integer value; subsequently, these integer flags determine which material property values to use in DEM systems with multiple types of minerals, e.g., particles flagged with the integer 255 are assigned mechanical properties consistent with a stiff material 
while flagging a particle with the integer 0 will assign the particle the properties of a more flexible material in this case. The choice of sizes of particles is described in Section 3.1, below.
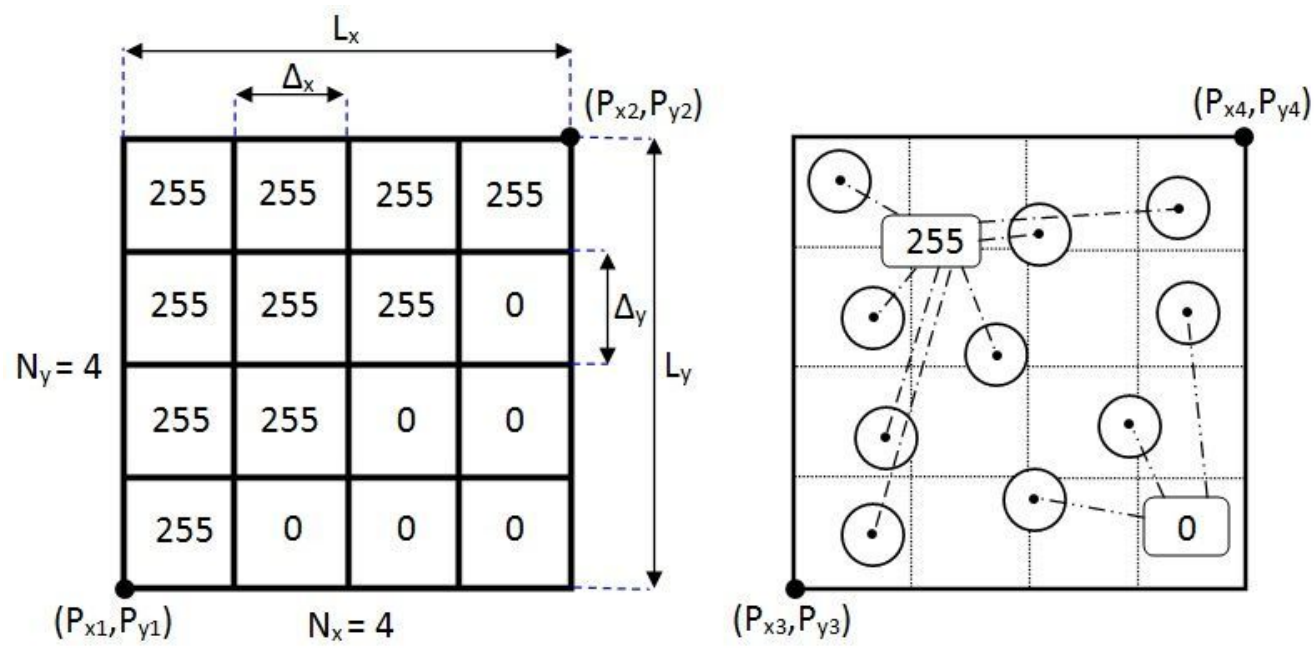

Figure 2: Example of assigning property identifications to particles based on their location with respect to an image in text file format superimposed over the particle system.

\subsection{DEM Particle Bonding Algorithms}

After the particles in a system are generated and settled in a manner similar to the work in [5], and properties are assigned, bonds are then generated between particles using algorithms based on the bonded-particle model also documented in [5]. The parallel bond model in [5] incorporates both normal contact and force transmission via bonds in the equations of motion associated with the particles. The equations of motion for the particles in these simulations are therefore related to only normal and shear forces and moments transmitted through the inter-particle bonds.

A bond between two particles, $i$ and $j$, as shown in Figure 3, is approximated by a beam of unit thickness, i.e. $t^{b}=1$, of length equal to the sum of the two particle radii, $L^{b}=R_{i}+R_{j}$, and having a cross sectional area of $A^{b}$, where the relation $A^{b}=2 \lambda \operatorname{Min}\left(R_{i}, R_{j}\right)$ is unit thickness times twice the bond radius, $R_{\lambda}=\lambda \operatorname{Min}\left(R_{i}, R_{j}\right)$; the determination of the bond radius $R_{\lambda}$ using the user-defined parameter $\lambda$ is described further in [5], and the effects on DEM system properties due to perturbations about $\lambda=1.0$ are shown in 


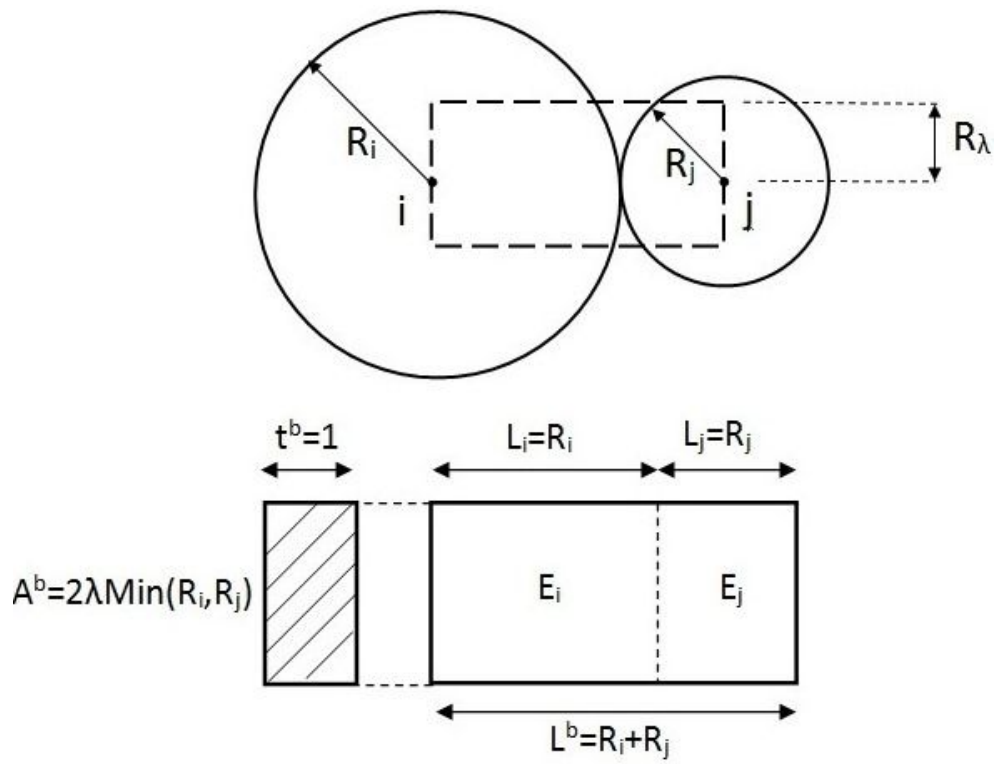

Figure 3: Geometry of a bond connecting particles $i$ and $j . A, E, L, \lambda, R$, and $t$ denote area, Young's modulus, length, a multiplier, radius, and thickness, respectively.

[27]. In this research, the multiplier is fixed to a value of $\lambda=1.0$. The superscript, $b$, indicates that the property is related to that of a particle bond. The two particles associated with a single bond in a particle system, $i$ and $j$, may have different radii and Young's moduli, thus necessitating incorporation of the function $\operatorname{Min}\left(R_{i}, R_{j}\right)$ in addition to determining the effective properties of the composite beam when $E_{i} \neq E_{j}$. As the bonds may transmit shear and normal loads, in addition to moments, and due to the fact that two particles of different material may be bonded together, the stiffness values must be approximated as two springs in series:

$$
\frac{1}{k_{n}^{b}}=\frac{1}{k_{i}}+\frac{1}{k_{j}}
$$

where $k_{n}^{b}$ is the normal bond stiffness, denoted by $n$, and $k_{i}=\frac{E_{i} A^{b}}{L_{i}}, k_{j}=\frac{E_{j} A^{b}}{L_{j}}$, and where $L_{i}=R_{i}$ and $L_{j}=R_{j}$. Utilizing appropriate substitutions and manipulations, (2) yields the following relation for the axial stiffness of the bond between particles $i$ and $j$ :

$$
k_{n}^{b}=\frac{E_{i} E_{j} A^{b}}{R_{i} E_{j}+R_{j} E_{i}} .
$$


It should be noted here that $E_{i}, E_{j}$ are the Young's moduli of the elastic bond between particles $i$ and $j$; $E_{\text {micro }}$ will refer to the same value, but it is used in the context of a microscopic parameter that must be calibrated in order to achieve desired macroscopic properties for a given mineral that is to be represented by a region of bonded DEM particles.

With regards to the shear stiffness, [5] relate the shear stiffness of a bond to its axial stiffness, eqn. (3), in a manner similar to the following equation:

$$
k_{s}^{b}=\beta k_{n}^{b},
$$

where $\beta$ is the ratio of shear bond stiffness to normal bond stiffness, i.e. $\beta=$ $\frac{k_{s}^{b}}{k_{n}^{b}}$. This ratio is described by [5] as having an influence on the macroscopic Young's modulus and Poisson's ratio of the system of bonded particles.

The bond shown in Figure 3 is assumed to have three deformation modes: axial, shear, and bending. Bending stiffness resists rotation of the ends of the bond relative to each other and is given by

$$
k_{t}^{b}=\frac{E_{i} E_{j} I^{b}}{R_{i} E_{j}+R_{j} E_{i}},
$$

where $I^{b}$ is the area moment of inertia and is defined as $I^{b}=\frac{1}{12} t^{b}\left(2 R_{\lambda}\right)^{3}=$ $\frac{2}{3} t^{b} R_{\lambda}^{3}$.

As will be demonstrated later in this paper, the relationships between the microscopic parameter $\beta$ from eqn. (4), and $E_{i}, E_{j}$ from eqn. (3), are used in calibrating the DEM systems so as to exhibit desired macroscopic behavior, i.e., macroscopic Young's modulus and Poisson's ratio.

\section{Homogenization via DEM}

\subsection{DEM-Based Estimates of Mineral Properties}

Three oil-shale samples, measuring approximately $20 \mathrm{~mm}$ on edge, were imaged, each generating an associated configuration for use in DEM, with the methodology as described in the previous section. Figure 4 shows three falsecolor images generated via the QEMSCAN process containing the geometric distribution and mineralogical data for the three samples.

The data shown in Figure 4 is used to assign the stiffness properties of DEM systems. The images are integrated into DEM simulations using the process discussed in Section 2. The particle sizes are of a rectangular distribution, or a continuous uniform distribution, with a mean of approximately 


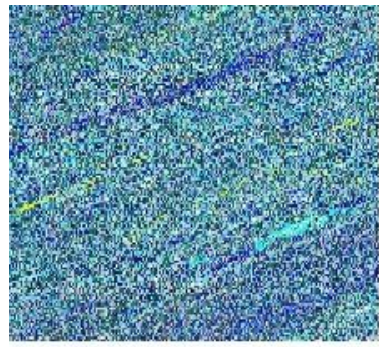

(1)

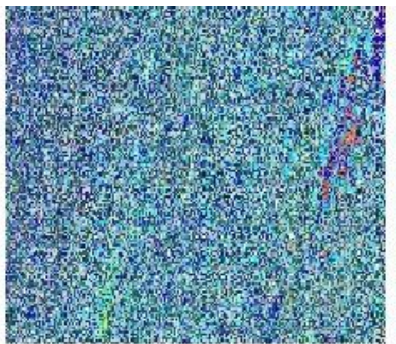

(II)

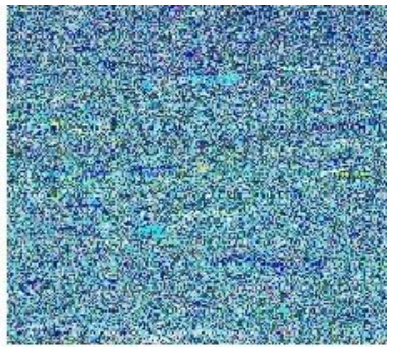

(III)

Figure 4: Images of the three samples of oil shale obtained through QEMSCAN corresponding to Samples I, II, and III. Each of the images represent a physical sample size of $400 \mathrm{~mm}^{2}$.

$0.156 \mathrm{~mm}$. The selection of the mean particle size was guided by an iterative process in which an arbitrary number of particles were generated and integrated with data from the images of geomaterials, as is discussed in Section 2. The mean particle size is the size at which the bedding planes seen in the geomaterial images, e.g. Figure 4, are distinguishable in the DEM representations of the images. While the mean particle size of $0.156 \mathrm{~mm}$ was used, the rectangular distribution about the mean was set so as to give the ratio of the radii of the largest particles to the smallest particles a value of $1.75: 1$; while an extensive investigation into the effects of increasing/decreasing this ratio was not performed in this work, the ratio of $1.75: 1$ was beneficial in reducing the number of grains forming among the particles in the DEM simulations.

The colors in the images identify the mineral in the samples, as well as their location on the scanned surfaces. Fourteen distinct minerals can be identified from the samples; however, several of the minerals are sparse enough such that their contributions to the mechanical behavior of a sample, as a whole, are considered to be negligible. Additionally, the mechanical properties of some minerals are so similar as to be considered the same mineral. Further details on the method of reduction of the number of minerals represented in the simulations can be found in [27]. With these assumptions, the 14 mineral types in the samples are reduced to 6 mineral types, and the adjusted area percentages of the minerals in each of the three samples can be found in Table 1 .

Microscopic parameters such as particle size distribution and packing of DEM particles can significantly alter the macroscopic elastic properties of DEM systems. The Young's moduli, E, and normal-to-shear stiffness ratios, 
Table 1: Percent areas of the samples containing 6 minerals after processing of the data seen in Figure 4.

\begin{tabular}{|c|c|c|c|}
\hline Material & Sample I & Sample II & Sample III \\
\hline Pyrite & 1.17 & 0.92 & 0.90 \\
\hline Feldspar & 20.62 & 18.90 & 17.38 \\
\hline Quartz & 24.21 & 24.54 & 23.11 \\
\hline Clay & 21.67 & 21.38 & 21.11 \\
\hline Dolomite & 22.41 & 24.63 & 26.39 \\
\hline Calcite & 9.92 & 9.63 & 11.11 \\
\hline
\end{tabular}

$\beta$ associated with the bonds, expressed in eqns. (3), (4), and (5) significantly affect estimates of the effective macroscopic Young's moduli and Poisson's ratios of the bonded DEM systems. In order to create bonded DEM systems with realistic mechanical properties to match those of the samples represented in Figure 4, the microparameters $E$ and $\beta$ are calibrated by measuring the stress-strain behavior of particle systems for each of the minerals shown in Table 1.

For the sake of clarity in the remainder of this section, there are three contexts in relation to the mechanical properties discussed. The first context is related to material properties found in the literature. As a benchmark for mineral properties by which to compare the methods described in this work, we refer to elastic properties found in [29], [30], [31], [32], [33], and [34]. Due to the variability in properties among the cited sources, the values from the aforementioned sources are averaged and set as the target values for parameter calibration in this research. Properties related to the benchmark mineral properties are henceforth denoted with the subscript, "lit". The second and third contexts are in relation to the bond properties and the properties of a DEM system representative of a single mineral type, denoted by subscripts "micro" and "macro," respectively.

The method used to estimate the bond properties needed to facilitate realistic Young's moduli and Poisson's ratios for each mineral type first involves recording the pre- and post-load coordinates of particles in a system. Particle centroid locations for an interior, rectangular region of several hundred particles, not including those near the boundaries of the systems, are 
recorded for analysis. In linear elasticity, strain is defined as

$$
\epsilon_{i j}=\frac{1}{2}\left(\frac{\partial u_{i}}{\partial x_{j}}+\frac{\partial u_{j}}{\partial x_{i}}\right),
$$

where $u_{i}$ is the $i$ th component of displacement and $i, j=1,2$ in plane stress and recall that $\nu=-\epsilon_{11} / \epsilon_{22}$. The one-dimensional form of Hooke's Law, associated with extension in the $x_{1}$ direction, i.e. $\sigma_{11}=E \epsilon_{11}$, can be rewritten as

$$
\frac{F_{\text {app }}}{A}=E \epsilon_{11},
$$

where $F_{\text {app }}$ is the force applied to the system, as shown in Figure 5; the strain in eqn. (7) is the normal strain in the $x_{1}$-direction. In the case of a continuum represented by a DEM system of particles, eqn.(7) becomes, after rearrangement to solve for the macroscopic estimate of the DEM system's Young's modulus,

$$
E_{\text {macro }}=\frac{F_{\text {app }}}{A_{\text {macro }}\left\langle\epsilon_{11}\right\rangle},
$$

where we have introduced the body average of the strain over the domain $R$ (with volume $|R|$ ) of the rectangular sample of the mineral in question, via angled brackets:

$$
\left\langle\epsilon_{11}\right\rangle=\frac{1}{|R|} \int_{R} \epsilon_{11} d V
$$

Furthermore $A_{\text {macro }}=t^{b} L_{2}$ and $t^{b}=1$, assuming the particle system and bonds are of unit thickness, and where $L_{x_{1}}, L_{x_{2}}$, and $F_{\text {app }}$ are the dimensions and applied load shown in Figure 5, respectively. Similar to eqn. (8), the macroscopic estimate of the DEM system's Poisson's ratio is

$$
\nu_{\text {macro }}=\frac{-\left\langle\epsilon_{22}\right\rangle}{\left\langle\epsilon_{11}\right\rangle}
$$

where $\left\langle\epsilon_{11}\right\rangle$ and $\left\langle\epsilon_{22}\right\rangle$ are averages of the particle systems' strains in the $x_{1}$ and $x_{2}$-directions.

The strains needed to calculate the macroscopic properties, i.e. eqns.(10) and (8), are found through the use of an approach similar to [35]. The displacements selected are calculated by subtracting the post-load particle coordinates from the pre-load coordinates; the boundary particles' displacements 
are excluded from these analyses so as to prevent inconsistencies in the strain estimates. To find an equation for a line whose slope is an estimate, $\left\langle\epsilon_{11}\right\rangle$, the particles' displacements with respect to their original $x_{1}$-coordinates are plotted versus the length of the sample. Basic equation-fitting algorithms yield an equation for a line of the form $y=m x+b$, where the slope of the line, $m$, is an estimate of the partial derivative of the particles' $x_{1}$-displacements with respect to the $x_{1}$-direction, i.e. $\left\langle\epsilon_{11}\right\rangle$. The same process can be used using the particles' $x_{2}$-displacements. While other measures have been proposed to calculate strain from DEM simulations, e.g. [36], [37], [10], and [38]. Various strain measures have been compared by [39].

The "macro" properties in Table 2 are obtained by first generating, settling, and bonding a rectangular, RP system of approximately 4, 400 particles with a maximum-to-minimum particle radius ratio, $R_{\max }: R_{\min }$, of $1.75: 1$. Stiffness properties are assigned to the bonds in the system by picking values for $E_{\text {micro }}$ and $\beta$, which are then integrated into the DEM algorithms through the use of eqns. (3), (4), and (5). Boundary conditions are imposed on the rectangular DEM model systems, and the response is observed so as to provide the estimates of $E_{\text {macro }}$ and $\nu_{\text {macro }}$ shown in Table 2.

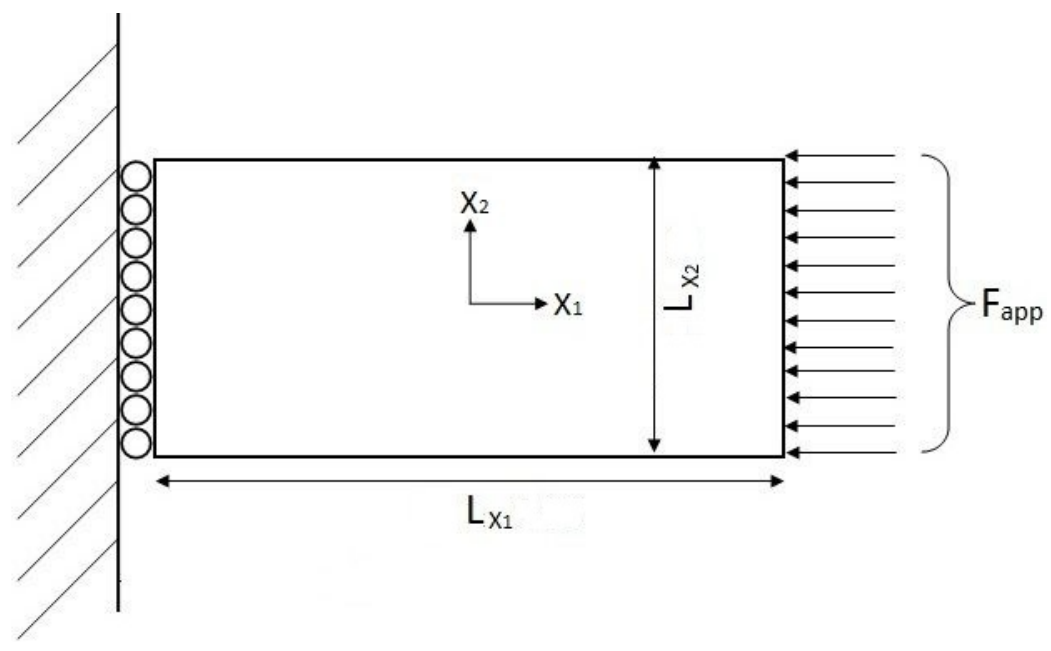

Figure 5: Diagram of a mechanical test with a compressive load.

The properties of the minerals shown in Table 1 are related to the stiffness of bonds in the DEM systems by eqns. (3), (4), and (5), and are listed in Table 2. The subscripts, "micro" and "macro," indicate the properties of the bonds and the macroscopic properties of the entire particle systems (the 
mineral phases here), respectively. For clarification, the "macro" values are those found through the initial stress/strain curve. The calibrations are considered to be successful when the values of $E_{\text {macro }}$ and $\nu_{\text {macro }}$ are within approximately $\pm 10 \%$ of $E_{\text {lit }}$ and $\nu_{\text {lit }}$.

Table 2: Comparison of benchpark properties $E_{\text {lit }}$ and $\nu_{\text {lit }}$, DEM parameters $E_{\text {micro }}$ and $\beta_{\text {micro }}$, and the macroscopic properties $E_{\text {macro }}$ and $\nu_{\text {macro }}$ for each material in the 6-mineral geomaterial simulations. Units for Young's moduli are in GPa.

\begin{tabular}{|c|c|c|c|c|c|c|}
\hline Material & $E_{\text {lit }}$ & $\nu_{\text {lit }}$ & $E_{\text {micro }}$ & $\beta_{\text {micro }}$ & $E_{\text {macro }}$ & $\nu_{\text {macro }}$ \\
\hline Pyrite & 305.90 & 0.154 & 305.90 & 0.518 & 306.38 & 0.163 \\
\hline Feldspar & 70.02 & 0.347 & 82.96 & 0.207 & 68.85 & 0.312 \\
\hline Quartz & 90.15 & 0.099 & 91.57 & 0.687 & 97.74 & 0.108 \\
\hline I-S & 45.79 & 0.286 & 50.32 & 0.280 & 44.30 & 0.269 \\
\hline Dolomite & 122.36 & 0.262 & 131.20 & 0.315 & 118.13 & 0.250 \\
\hline Calcite & 73.41 & 0.335 & 85.56 & 0.220 & 71.77 & 0.304 \\
\hline
\end{tabular}

\subsection{Homogenization Process}

Having determined the effective properties of each separate mineral phase via the homogenization scheme described above, we now turn our attention to estimating the effective macroscopic properties of each of the three inhomogeneous samples in question. The calibration process involved particle systems of approximately 4, 400 particles and a length-to-width ratio of $3: 1$. The coordination number for the DEM systems used in the calibration process is 4.65. Larger particle systems are created using the process described in Section 2.2, and the same particle size distribution is used as during the microparameter calibrations; the larger systems, however, have an overall length-to-width ratio of $1: 1$ so as to facilitate straightforward integration with the square geomaterial images. The coordination number for the larger DEM systems is 4.91. All DEM samples have an initial porosity of $9.55 \%$. The images in Figure 4 are used to assign material identities to the particles, and the information in Table 2 is used to give appropriate stiffness properties to the particle bonds based on their mineralogical representations. A biharmonic average of the properties shown in Table 2 is used to determine the stiffness of bonds connecting two particles with different assigned material identities. Similar to the methods used to calibrate the microparameters for the individual mineral types, boundary conditions are applied to other particle systems, and the pre- and post-load locations of interior particles in 
the systems are recorded. Since the images in Figure 4 obviously indicate bedding planes in the samples, two different loading scenarios are used for each of the three geomaterial images; the differences between the estimated properties for one loading scenario over the other provides interesting information with regards to the in-plane anisotropy of the samples. Figure 6 shows a sketch of the two loading scenarios. Eqns. (8) and (10) are used to determine the in-plane Young's moduli and Poisson's ratios for the three samples, for both of the loading scenarios shown in Figure 6.

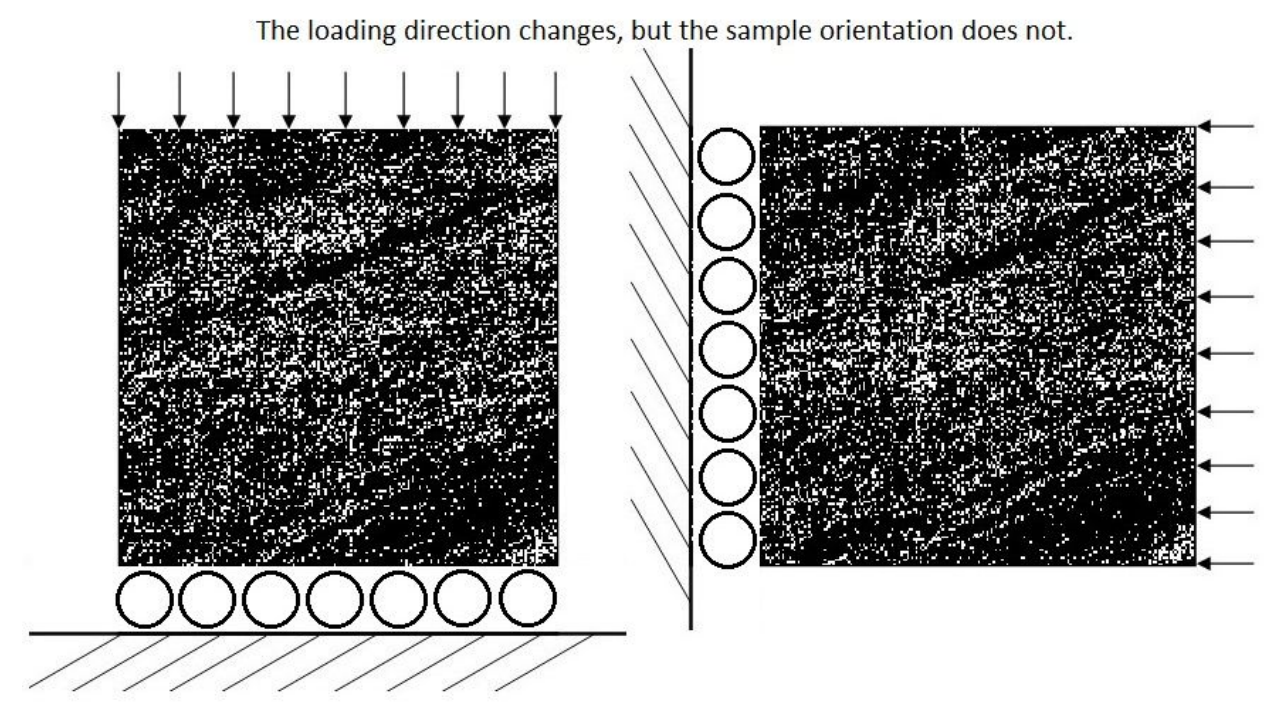

Figure 6: Sketch of the loading direction with respect to the images used in the DEM analyses. The vertical (left) and horizontal (right) loadings are applied to DEM systems whose particles have been assigned properties based on the contents of Figure 4.

\section{Homogenization via Micromechanical Methods}

\subsection{The Polycrystalline Self-Consistent Method}

All micromechanical methods begin with the standard form of Hooke's law for inhomogeneous anisotropic media:

$$
\sigma_{i j}(\boldsymbol{x})=C_{i j k l}(\boldsymbol{x}) \epsilon_{k l}(\boldsymbol{x}),
$$


where $\sigma_{i j}$ and $C_{i j k l}$ are the stress and elastic modulus tensors respectively and $\epsilon_{k l}$ is the strain tensor introduced in (6). The aim is to replace (11) with a homogeneous equivalent medium with effective elastic modulus tensor $C_{i j k \ell}^{*}$ in the RVE, so that we can write

$$
\left\langle\sigma_{i j}\right\rangle=C_{i j k l}^{*}\left\langle\epsilon_{k l}\right\rangle
$$

where angled brackets denote the volume average over the domain $V$ of inhomogeneous material, in a similar manner to (9), i.e.

$$
\left\langle\sigma_{i j}\right\rangle=\frac{1}{|V|} \int_{V} \sigma_{i j}(\boldsymbol{x}) \mathrm{d} \boldsymbol{x}
$$

where $|V|$ denotes the volume of the domain $V$. In Figure 7, the region $D$ denotes the matrix phase in a heterogeneous material while regions $\Omega$ denotes inclusion phases. The domain $V$ is simply the contiguous matrix/inclusion system, or $V=D \cup \Omega$.
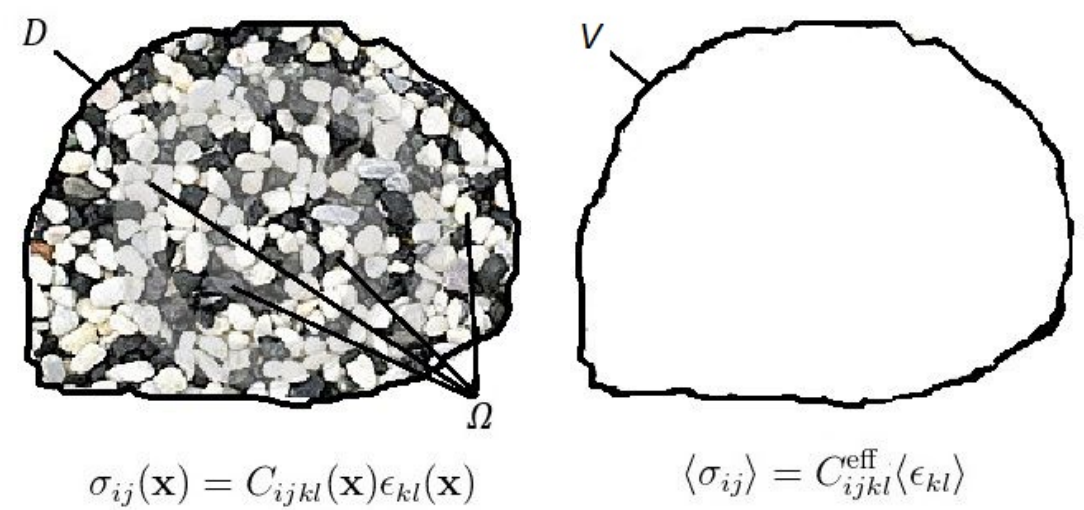

Figure 7: Visualization of Eqn. (11) and Eqn. (12). $D$ denotes the matrix phase of a heterogeneous material, and $\Omega$ denotes the inclusion phases. $V$ includes both matrix and inclusion phases.

Next define the volume averaged strain over the domain $V_{r}$ of the $r$ th phase as

$$
\left\langle\epsilon_{i j}\right\rangle^{r}=\frac{1}{\left|V_{r}\right|} \int_{V_{r}} \epsilon_{i j}(\boldsymbol{x}) \mathrm{d} \boldsymbol{x},
$$

where $\left|V_{r}\right|$ indicates the volume of the domain $V_{r}$. By introducing the strain concentration tensor linking $\left\langle\epsilon_{i j}\right\rangle^{r}$ to the overall volume averaged strain $\left\langle\epsilon_{i j}\right\rangle$, 
i.e.

$$
\left\langle\epsilon_{i j}\right\rangle^{r}=A_{i j k \ell}\left\langle\epsilon_{k \ell}\right\rangle
$$

it is straightforward to show that [3]

$$
C_{i j k \ell}^{*}=C_{i j k \ell}^{0}+\sum_{r=1}^{N} \phi_{r}\left(C_{i j k \ell}^{r}-C_{i j k \ell}^{0}\right) A_{i j k \ell}^{r},
$$

in the case when a discernible host phase (phase 0 here) is present and where the volume fraction $\phi_{r}=\left|V_{r}\right| /|V|$. When there is no discernible host phase, i.e. for polycrystalline media, the relevant expression is instead

$$
C_{i j k \ell}^{*}=\sum_{r=1}^{N} \phi_{r} C_{i j k \ell}^{r} A_{i j k \ell}^{r} .
$$

If $A_{i j k \ell}^{r}$ is calculated exactly here then these expressions will give the correct effective properties. The difficulty resides, however, in determining the concentration tensor since in general a particle of phase $r$ will feel the presence of all particles surrounding it and a careful numerical simulation is therefore required. Micromechanical methods utilize a number of ingenious approximations in order to estimate the components of the concentration tensor. The most simple (and naive) of these is the dilute estimate, which uses the strain concentration tensor that arises when an inhomogeneity associated with phase $r$ is isolated, e.g. $A_{i j k \ell}^{r}=\mathcal{A}_{i j k \ell}^{r}$ [14] so that it does not feel the presence of other inhomogeneities. This is therefore only valid for rather small volume fractions.

Geomaterials can be regarded as polycrystalline and therefore it appears appropriate to employ (17). A method that has become very popular for polycrystalline media, is to estimate $A_{i j k \ell}^{r}$ as the strain concentration tensor associated with an isolated particle of the $r$ th phase embedded in the effective (i.e. unknown) medium. We therefore write this as $A_{i j k \ell}^{r} \approx \mathcal{A}_{i j k \ell}^{r *}$. Using this in (17) means that it becomes an implicit equation for the effective properties. In general what arises is a system of nonlinear equations that needs to be solved numerically for the determination of the effective properties and those that are physical are retained as the predicted property; solutions to the nonlinear system of equations having aphysical values, such as a negative Young's modulus, are rejected. Using the PSCM, the general expression 
for the strain concentration tensor associated with the $r$ th phase can be conveniently written in terms of the components of its inverse: $\tilde{\mathcal{A}}_{i j k \ell}^{r *}$ via

$$
\tilde{\mathcal{A}}_{i j k \ell}^{r *}=I_{i j k \ell}+P_{i j m n}^{r *}\left(C_{m n k \ell}^{r}-C_{m n k \ell}^{*}\right), \quad A_{i j m n}^{r *} \tilde{A}_{m n k \ell}^{r *}=I_{i j k \ell}
$$

where $I_{i j k \ell}=\left(\delta_{i k} \delta_{j \ell}+\delta_{i \ell} \delta_{j k}\right) / 2$ is the fourth order identity tensor and $P_{i j k \ell}^{r}$ is Hill's tensor associated with the particle of phase $r$ embedded in the effective medium. Recall that Hill's tensor only depends on the shape of the $r$ th phase and not its elastic properties. It can be evaluated analytically for a large class of ellipsoidal inhomogeneities [14]. In particular, the components for Hill's tensor for circular cylindrical inhomogeneities embedded in an transversely isotropic host phase is given in (5.153)-(5.154) of [14]. This is appropriate here since we assume here that the effective elastic modulus tensor is transversely isotropic.

Note again here that inhomogeneities are assumed to be circular cylinders with the axis of rotational symmetry aligned with the axis that is perpendicular to the plane of the SEM images.

\subsection{Homogenization process}

The same target values used for the DEM parameter calibrations, i.e. $E_{\text {lit }}$ and $\nu_{\text {lit }}$ in Table 2 , in addition to the volume fraction data shown in Table 1 , are used in eqn. (17) in order to fully populate the effective stiffness tensors representative of the three geomaterial images processed using QEMSCAN. Due to assumptions stated earlier in this work with regards to the shape and alignment of the circular, cylindrical inclusions used in the application of the PSCM, the elastic properties gleaned from this homogenization technique are considered to be relevant to a plane strain condition. Further justification for the plane strain assumption stems from the fact that the images seen in Figure 4 tell nothing of the samples' properties out of the plane of the images. As such, the properties reported for a homogenization-based analysis of the geomaterials should be considered to be with respect to the plane of the geomaterial images.

\section{Results \& Discussion}

Since the 2D DEM systems in this work have out-of-plane bonds and particles of unit thickness, and the in-plane dimensions of both the 4,400and 16,500-particle systems are much greater than unity, the results of the 
DEM analyses are considered to be appropriate for plane stress conditions. In order to compare the DEM-based effective properties to those of the PSCMbased analyses, the homogenization results are converted from plane strain to plane stress via the expressions

$$
E_{\sigma}=\frac{E_{\epsilon}\left(1+2 \nu_{\epsilon}\right)}{\left(1+\nu_{\epsilon}\right)^{2}}
$$

and

$$
\nu_{\sigma}=\frac{\nu_{\epsilon}}{1+\nu_{\epsilon}}
$$

where the subscripts $\sigma$ and $\epsilon$ indicate a state of plane stress and plane strain, respectively.

The results of the DEM- and homogenization-based approaches to estimating the effective elastic properties of the three geomaterial samples are given in Table 3. Subscripts such as "mv" or "mh" indicate the macroscopic results from DEM analyses and their vertical or horizontal loading directions, respectively, and subscripts such as "PSCM- $\sigma$ " indicate the homogenization results converted to plane stress.

Table 3: Results of the 6-mineral geomaterial samples calculated using PSCM and DEM. The effective values calculated using PSCM have been converted to plane stress. Young's moduli are in units of GPa.

\begin{tabular}{|c|c|c|c|c|c|c|}
\hline Sample & $E_{\mathrm{PSCM}-\sigma}$ & $E_{\mathrm{mv}}$ & $E_{\mathrm{mh}}$ & $\nu_{\mathrm{PSCM}-\sigma}$ & $\nu_{\mathrm{mv}}$ & $\nu_{\mathrm{mh}}$ \\
\hline I-M & 74.74 & 76.17 & 75.62 & 0.208 & 0.228 & 0.227 \\
\hline II-M & 75.59 & 76.10 & 76.51 & 0.207 & 0.226 & 0.227 \\
\hline III-M & 76.00 & 77.21 & 76.07 & 0.208 & 0.228 & 0.227 \\
\hline
\end{tabular}

The results in Table 3 show good agreement between the estimates of Young's moduli using PSCM and DEM. In particular the biggest discrepancy between the methods occurs in Sample I-M at 1.90\%. The largest difference in the Poisson's ratios occurs for Sample II-M at 9.22\%. It should be noted that the data shown in Table 3 is qualitative rather than quantitative. A qualitative analysis of geomaterials using DEM- and PSCM-based approaches would require the inclusion of void and organic content information, which is beyond the scope of this work.

Both DEM and PSCM appear to provide consistent estimates for the effective properties of these highly heterogeneous geomaterials; that said, there is clearly more information regarding the nature of the materials present in 
the DEM simulations. The assumptions made about inclusion shapes in the PSCM in this work leads to transversely isotropic effective properties. The Young's moduli calculated using PSCM, presented in Table 3, are in reference to the stiffness in the plane of the geomaterial images. The Poisson's ratios obtained through the use of the PSCM merely show a relationship between the in-plane and out-of-plane strains and no information regarding a relationship between the two in-plane cardinal directions, while visual inspection of Figure 4 shows layering that would cause in-plane anisotropy in the materials.

If one considers the contents of the DEM-based results in Table 3, the sketch in Figure 4, and the images in Figure 6, it can be seen that the materials are found to be stiffer when the loading directions are more closely aligned with the bedding planes and less stiff when loaded perpendicular to the planes. This is intuitively consistent with a simple parallel/series spring problem; two springs connected in parallel between a fixed and a moving object provide greater resistance to stretching or compression than two springs in series.

The slight differences between the magnitudes of the Young's moduli between the two methods of analysis show that either method might be used as an indication that the other is producing reasonable results. That said, the two methods do differ in terms of speed of computation and the types of anisotropy that can be detected in the results. For the PSCM, only the volume fractions and mineral identities must be known for an estimate of mechanical properties which, in this work, requires a few moments of computational time on a standard desktop or laptop computer. The DEMbased method provides Young's moduli and relevant in-plane Poisson's ratios (in other words, an indication of in-plane anisotropy), but it also requires much more time invested in computation and the DEM system setup. In the DEM simulations in this work, the generation, bonding, and settling process before the geomaterial image data is incorporated in the model required about thirty minutes to an hour on a standard desktop or laptop.

\section{Conclusion}

Data from images obtained using scanning acoustic microscopy, in conjunction with the discrete-element-based and PSCM-based methods discussed in this work, have been utilized to provide estimates of the effective elastic moduli of the geomaterials'; however, while both property estimation 
methods' results were reasonably close to each other, the DEM results contained information on the anisotropic nature of the samples while the PSCM method did not due to assumptions made regarding the shapes of the inclusions. Scans illustrate that a large number of minerals are present, and for the sake of computation these were restricted to six types, which was felt to be sufficient to accurately represent the media in question. The PSCM homogenization technique was used, under the assumption of circular cylindrical inclusions to represent the heterogeneities in the geomaterials, as the characteristics of the shale samples out of the plane of the images are considered to be unknown. The assumption of circular, cylindrical inclusions causes the effective stiffness tensors of the homogenized materials to be transversely isotropic, suggesting that the visually-noticeable bedding planes in the geomaterial images result in no directionally-dependent changes in elastic properties. An assumption of elliptical, cylindrical inclusions would provide a way to introduce in-plane directional dependence into the effective elastic properties (orthotropy instead of transverse isotropy) of these heterogeneous materials with clear structural anisotropy. Future work is suggested in exploring PSCM-based homogenization to DEM with elliptical, cylindrical inclusions assumed for the former.

Another issue with the DEM analyses presented in this research is the current inability to predict microscopic bond stresses from macroscopic applied stresses in RP systems. A suggested subject for future work is to compute the gradients of displacement fields in localized regions located along a cross section of a DEM system that is perpendicular to the loading direction. Using Hooke's law for the strains calculated in each of these displacement fields would yield estimates for the stress state in each of the localized regions. The stress state estimates would then be compared to the macroscopic applied stress.

\section{Acknowledgements}

SG, JRB and GGWM would like to acknowledge the support of the Center for Oil Shale Technology and Research (COSTAR) at the Colorado School of Mines. WJP is grateful to the Engineering and Physical Sciences Research Council UK for funding his research fellowship (EP/L018039/1). 


\section{References}

[1] B. Klusemann, B. Svendsen, Homogenization methods for multi-phase elastic composites: Comparisons and benchmarks, Technische Mechanik 30 (4) (2010) 374-386.

[2] J. Qu, M. Cherkaoui, Fundamentals of Micromechanics of Solids, Wiley, 2006.

[3] G. Dvorak, Micromechanics of composite materials, Vol. 186, Springer Science \& Business Media, 2012.

[4] Y. Capdeville, M. Zhao, P. Cupillard, FastFourier homogenization for elastic wave propagation in complex media, Wave Motion 54 (2015) 170-186.

[5] D. Potyondy, P. Cundall, A bonded-particle model for rock, International journal of rock mechanics and mining sciences 41 (8) (2004) 13291364 .

[6] H.-G. Matuttis, J. Chen, Understanding the Discrete Element Method: Simulation of Non-spherical Particles for Granular and Multi-body Systems, John Wiley \& Sons, 2014.

[7] S. R. Buechler, J. R. Berger, G. G. Mustoe, A discrete element approach to elastic properties of layered geomaterials, International Journal for Numerical and Analytical Methods in Geomechanics 37 (7) (2013) 685700 .

[8] C. S. Chang, C. L. Liao, Estimates of elastic modulus for media of randomly packed granules, Applied Mechanics Reviews 47 (1S) (1994) S197-S206.

[9] V. Magnanimo, L. La Ragione, J. Jenkins, P. Wang, H. Makse, Characterizing the shear and bulk moduli of an idealized granular material, EPL (Europhysics Letters) 81 (3) (2008) 34006.

[10] R. Bathurst, L. Rothenburg, Micromechanical aspects of isotropic granular assemblies with linear contact interactions, Journal of Applied Mechanics 55 (1) (1988) 17-23. 
[11] E. Somfai, J.-N. Roux, J. H. Snoeijer, M. Van Hecke, W. Van Saarloos, Elastic wave propagation in confined granular systems, Physical Review E 72 (2) (2005) 021301.

[12] W. Voigt, Ueber die beziehung zwischen den beiden elasticitätsconstanten isotroper körper, Annalen der Physik 274 (12) (1889) 573-587.

[13] A. Reuss, Berechnung der fließgrenze von mischkristallen auf grund der plastizitätsbedingung für einkristalle., ZAMM-Journal of Applied Mathematics and Mechanics/Zeitschrift für Angewandte Mathematik und Mechanik 9 (1) (1929) 49-58.

[14] W. J. Parnell, The Eshelby, Hill, moment and concentration tensors for ellipsoidal inhomogeneities in the Newtonian potential problem and linear elastostatics, Journal of Elasticity (2016) 1-64.

[15] J. D. Eshelby, The determination of the elastic field of an ellipsoidal inclusion, and related problems, in: Proceedings of the Royal Society of London A: Mathematical, Physical and Engineering Sciences, Vol. 241, The Royal Society, 1957, pp. 376-396.

[16] Y. Benveniste, A new approach to the application of Mori-Tanaka's theory in composite materials, Mechanics of materials 6 (2) (1987) 147157.

[17] A. Norris, A differential scheme for the effective moduli of composites, Mechanics of materials 4 (1) (1985) 1-16.

[18] R. Hill, A self-consistent mechanics of composite materials, Journal of the Mechanics and Physics of Solids 13 (4) (1965) 213-222.

[19] B. Budiansky, On the elastic moduli of some heterogeneous materials, Journal of the Mechanics and Physics of Solids 13 (4) (1965) 223-227.

[20] S. Kanaun, V. Levin, Self-Consistent Methods for Composites: Vol. 1: Static Problems, Vol. 148, Springer Science \& Business Media, 2007.

[21] S. Kanaun, V. Levin, J. G. Berryman, Self-consistent methods for composites, volume 2 - wave propagation in heterogeneous materials, Acoustical Society of America Journal 125 (2009) 1256. 
[22] R. Christensen, K. Lo, Solutions for effective shear properties in three phase sphere and cylinder models, Journal of the Mechanics and Physics of Solids 27 (4) (1979) 315-330.

[23] J. Paiboon, D. Griffiths, J. Huang, G. A. Fenton, Numerical analysis of effective elastic properties of geomaterials containing voids using $3 \mathrm{~d}$ random fields and finite elements, International Journal of Solids and Structures 50 (20) (2013) 3233-3241.

[24] N. Willoughby, W. J. Parnell, A. L. Hazel, I. D. Abrahams, Homogenization methods to approximate the effective response of random fibrereinforced composites, International Journal of Solids and Structures 49 (13) (2012) 1421-1433.

[25] A. A. Gusev, Representative volume element size for elastic composites: a numerical study, Journal of the Mechanics and Physics of Solids 45 (9) (1997) 1449-1459.

[26] R. Pascoe, M. Power, B. Simpson, \{QEMSCAN\} analysis as a tool for improved understanding of gravity separator performance, Minerals Engineering 20 (5) (2007) 487 - 495, selected papers from Automated Mineralogy 06, Brisbane, Australia, July 2006. doi:http://dx.doi.org/10.1016/j.mineng.2006.12.012.

[27] S. Geer, Discrete Element and Homogenization Based Approaches for Determining the Mechanical Properties of Geomaterials, Ph.D. thesis, Colorado School of Mines, Golden (2014).

[28] M. D. Abràmoff, P. J. Magalhães, S. J. Ram, Image processing with Imagej, Biophotonics international 11 (7) (2004) 36-43.

[29] R. S. Carmichael, Physical properties of rocks and minerals, CRC, Boca Raton, Florida.

[30] E. Fjar, R. M. Holt, A. Raaen, R. Risnes, P. Horsrud, Petroleum related rock mechanics, Vol. 53, Elsevier, 2008.

[31] G. Mavko, T. Mukerji, J. Dvorkin, The rock physics handbook: Tools for seismic analysis in porous media, 329 pp (1998). 
[32] D. M. D. Jizba, Seismic measurements, Carbonate Seismology 6 (1997) 75 .

[33] L.-g. Liu, C.-c. Chen, C.-C. Lin, Y.-j. Yang, Elasticity of single-crystal aragonite by Brillouin spectroscopy, Physics and chemistry of minerals 32 (2) (2005) 97-102.

[34] Z. Wang, H. Wang, M. E. Cates, Effective elastic properties of solid clays, Geophysics 66 (2) (2001) 428-440.

[35] C.-L. Liao, T.-P. Chang, D.-H. Young, C. S. Chang, Stress-strain relationship for granular materials based on the hypothesis of best fit, International Journal of Solids and Structures 34 (31) (1997) 4087-4100.

[36] K. Bagi, Stress and strain in granular assemblies, Mechanics of materials 22 (3) (1996) 165-177.

[37] B. Cambou, M. Chaze, F. Dedecker, Change of scale in granular materials, European Journal of Mechanics-A/Solids 19 (6) (2000) 999-1014.

[38] M. R. Kuhn, Scaling in granular materials, Powders and grains 1 (2005) 115-122.

[39] K. Bagi, Analysis of microstructural strain tensors for granular assemblies, International Journal of Solids and Structures 43 (10) (2006) 31663184 . 